\title{
STABLE COHOMOLOGY OF ALTERNATING GROUPS
}

\author{
FEDOR BOGOMOLOV ${ }^{1}$ AND CHRISTIAN BÖHNING ${ }^{2}$
}

\begin{abstract}
In this article we determine the stable cohomology groups $H_{\mathrm{s}}^{i}\left(\mathfrak{A}_{n}, \mathbb{Z} / p \mathbb{Z}\right)$ of the alternating groups $\mathfrak{A}_{n}$ for all integers $n$ and $i$, and all primes $p$.
\end{abstract}

\section{INTRODUCTION AND PRELIMINARIES}

Let $G$ be a finite group, $V$ a finite-dimensional generically free complex representation of $G$, and let $V^{L} \subset V$ be the nonempty open subset of $V$ on which the $G$-action is free. There is then a natural homotopy class of maps from the classifying space $\mathrm{B} G$ to $V^{L} / G$ which, for each nonempty $G$-invariant Zariski open subset $U \subset V^{L}$ gives maps $H^{i}(G, \mathbb{Z} / p \mathbb{Z}) \rightarrow$ $H^{i}(U / G, \mathbb{Z} / p \mathbb{Z})$. It turns out that the kernel $K_{G, V}$ of

$$
H^{i}(G, \mathbb{Z} / p \mathbb{Z}) \rightarrow \underset{U}{\lim } H^{i}(U / G, \mathbb{Z} / p \mathbb{Z})
$$

is independent of $V$, and the stable cohomology $H_{\mathrm{s}}^{i}(G, \mathbb{Z} / p \mathbb{Z})$ is defined to be the quotient $H^{i}(G, \mathbb{Z} / p \mathbb{Z}) / K_{V, G}$. Let $K=\mathbb{C}(V)^{G}$. Algebraically, $H_{\mathrm{s}}^{i}(G, \mathbb{Z} / p \mathbb{Z})$ can be identified with the image of $H^{i}(G, \mathbb{Z} / p \mathbb{Z})$ in $H^{i}(\operatorname{Gal}(K), \mathbb{Z} / p \mathbb{Z})$.

More accessible computationally and stable birational invariants of the function field $K$ are the unramified cohomology groups $\mathrm{H}_{\mathrm{nr}}^{i}(G, \mathbb{Z} / p \mathbb{Z})$ defined as follows: geometrically, the unramified cohomology classes $a$ inside $\mathrm{H}_{\mathrm{s}}^{i}(G, \mathbb{Z} / p \mathbb{Z})$ are those for which, given any divisorial valuation $\nu_{D}$ of $K$, there exists a normal model $X=X_{D}$ of $K$ on which $\nu_{D}$ has a center, an isomorphism $i: U_{X} \rightarrow U_{V^{L} / G}$ between nonempty open subsets $U_{X}$ of $X$ and $U_{V^{L} / G}$ of $V^{L} / G$, and a representative $a^{\prime}$ of $a$ in $H^{i}\left(U_{V^{L} / G}, \mathbb{Z} / p \mathbb{Z}\right)$ such that there is a class $b \in H^{i}(X, \mathbb{Z} / p \mathbb{Z})$ whose image in $H^{i}\left(U_{X}, \mathbb{Z} / p \mathbb{Z}\right)$ coincides with $i^{*}\left(a^{\prime}\right)$. More algebraically, if $\mathcal{O}_{\nu} \subset K$ is the valuation ring of $\nu, \kappa_{\nu}=\mathcal{O}_{\nu} / \mathfrak{m}_{\nu}$ its residue field, $S=\operatorname{Spec}\left(\mathcal{O}_{\nu}\right)$ with open subset the generic point $U=\operatorname{Spec}(K) \subset S$

\footnotetext{
${ }^{1}$ Supported by NSF grant DMS-1001662 and by AG Laboratory GU- HSE grant RF government ag. 11 11.G34.31.0023.

${ }^{2}$ Supported by the German Research Foundation (Deutsche Forschungsgemeinschaft) through Heisenberg-Stipendium BO 3699/1-1.
} 
and complement the closed point $Z=\operatorname{Spec}\left(\kappa_{\nu}\right) \subset S$, one can write down the long exact sequence of étale cohomology with supports

$\cdots \rightarrow H^{i}(S, \mathbb{Z} / p) \rightarrow H^{i}(U, \mathbb{Z} / p) \rightarrow H_{Z}^{i+1}(S, \mathbb{Z} / p) \rightarrow H^{i+1}(S, \mathbb{Z} / p) \rightarrow \ldots$

where $H^{i}(U, \mathbb{Z} / p) \simeq H^{i}(\operatorname{Gal}(K), \mathbb{Z} / p)$ and there is the cohomological purity isomorphism

$$
H_{Z}^{j}(S, \mathbb{Z} / p) \simeq H^{j-2}(Z, \mathbb{Z} / p)
$$

whence the preceding sequence becomes the Gysin sequence

$$
\begin{gathered}
\cdots \rightarrow H_{\text {ét }}^{i}(S, \mathbb{Z} / p) \stackrel{r_{\nu}}{\rightarrow} H^{i}(\operatorname{Gal}(K), \mathbb{Z} / p) \\
\stackrel{\partial_{\nu}}{\rightarrow} H^{i-1}\left(\operatorname{Gal}\left(\kappa_{\nu}\right), \mathbb{Z} / p\right) \rightarrow H_{\text {ét }}^{i+1}(S, \mathbb{Z} / p) \rightarrow \ldots .
\end{gathered}
$$

A class in $H^{i}(\operatorname{Gal}(K), \mathbb{Z} / p)$ is clearly unramified according to the geometric definition if and only if it is in the image of all maps $r_{\nu}$ for $\nu$ running over the divisorial valuations of $K$, i.e. equivalently if it is in the kernel of all maps $\partial_{\nu}$, the residue maps. The preceding sequence has as topological analogue the Borel-Moore long exact sequence. The residue map

$$
\partial_{\nu}: H^{i}(\operatorname{Gal}(K), \mathbb{Z} / p) \rightarrow H^{i-1}\left(\operatorname{Gal}\left(\kappa_{\nu}\right), \mathbb{Z} / p\right)
$$

agrees -up to a sign- with the following map defined entirely within the framework of Galois cohomology (see e.g. GMS, Chap. II of Serre's part, $\S 6$ and $\S 7$ ): extend $\nu$ in some way to a valuation $\nu^{*}$ on $\bar{K}$ which is possible by Chevalley's theorem; all such extensions are conjugate under $\Gamma=\operatorname{Gal}(K)$, and $\nu^{*}$ defines subgroups $\Gamma_{Z} \subset \Gamma$ (the decomposition group, Zerlegungsgruppe) and $\Gamma_{T} \subset \Gamma$ (the inertia subgroup, Trägheitsgruppe) by the conditions that $\sigma \in \Gamma$ is in $\Gamma_{Z}$ if $\sigma \cdot \nu^{*}$ and $\nu^{*}$ are equivalent valuations, i.e. have the same valuation ring, and $\Gamma_{T}$ consists of those $\sigma$ such that $\sigma \cdot x-x \in \mathfrak{M}_{\nu^{*}}$ for all $x$ in the valuation ring of $\nu^{*}$ whose maximal ideal we denoted by $\mathfrak{M}_{\nu^{*}}$. The decomposition group can be identified with the Galois group $\operatorname{Gal}\left(\bar{K}_{\nu} / K_{\nu}\right)$ of the completion of $K$ with respect to $\nu$. The residue map $\partial_{\nu}$ then factors over the restriction to the decomposition group

$$
\partial_{\nu}: H^{i}(\operatorname{Gal}(K), \mathbb{Z} / p) \rightarrow H^{i}\left(\operatorname{Gal}\left(K_{\nu}\right), \mathbb{Z} / p\right) \stackrel{r}{\rightarrow} H^{i-1}\left(\operatorname{Gal}\left(\kappa_{\nu}\right), \mathbb{Z} / p\right)
$$

where the second arrow has the following description in the local situation: the Galois group $\Gamma_{K_{\nu}}=\operatorname{Gal}\left(K_{\nu}\right)$ sits in the exact sequence

$$
1 \rightarrow I \rightarrow \Gamma_{K_{\nu}} \rightarrow \Gamma_{\kappa_{\nu}}=\operatorname{Gal}\left(\kappa_{\nu}\right) \rightarrow 1
$$

where the surjection is given by the fact that $\nu$ extends uniquely to $\bar{K}_{\nu}$ and the residue field of the extension is an algebraic closure of $\kappa_{\nu}$. The kernel is the inertia subgroup which we denote by $I$ in this 


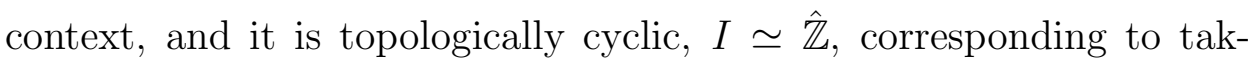
ing roots of the uniformizing parameter, and the preceding sequence splits, $\Gamma_{K_{\nu}} \simeq \hat{\mathbb{Z}} \oplus \operatorname{Gal}\left(\kappa_{\nu}\right)$. As $\hat{\mathbb{Z}}$ has cohomological dimension 1, one gets $H^{i}\left(\Gamma_{K_{\nu}}, \mathbb{Z} / p\right) \simeq H^{i}\left(\Gamma_{\kappa_{\nu}}, \mathbb{Z} / p \mathbb{Z}\right) \oplus H^{i-1}\left(\Gamma_{\kappa_{\nu}}, \mathbb{Z} / p \mathbb{Z}\right)$ and a projection, which is independent of the splitting, $H^{i}\left(\operatorname{Gal}\left(K_{\nu}\right), \mathbb{Z} / p\right) \stackrel{r}{\rightarrow}$ $H^{i-1}\left(\operatorname{Gal}\left(\kappa_{\nu}\right), \mathbb{Z} / p\right)$, defining the second arrow in the sequence of maps yielding $\partial_{\nu}$. More precisely, the Hochschild-Serre spectral sequence of the group extension of $\Gamma_{\kappa_{\nu}}$ by $I$

$$
H^{p}\left(\Gamma_{\kappa_{\nu}}, H^{q}(I, \mathbb{Z} / p)\right) \Longrightarrow H\left(\Gamma_{K_{\nu}}, \mathbb{Z} / p\right)
$$

reduces to a long exact sequence as $H^{i}(I, \mathbb{Z} / p)=0$ for $i \geq 2, H^{0}(I, \mathbb{Z} / p)=$ $\mathbb{Z} / p, H^{1}(I, \mathbb{Z} / p)=\operatorname{Hom}(I, \mathbb{Z} / p)=\mathbb{Z} / p$, which reads

$$
\begin{gathered}
\cdots \rightarrow H^{i}\left(\Gamma_{\kappa_{\nu}}, \mathbb{Z} / p\right) \rightarrow H^{i}\left(\Gamma_{K_{\nu}}, \mathbb{Z} / p\right) \rightarrow H^{i-1}\left(\Gamma_{\kappa_{\nu}}, \operatorname{Hom}(\hat{\mathbb{Z}}, \mathbb{Z} / p)\right) \rightarrow \\
\rightarrow H^{i+1}\left(\Gamma_{\kappa_{\nu}}, \mathbb{Z} / p\right) \rightarrow H^{i+1}\left(\Gamma_{K_{\nu}}, \mathbb{Z} / p\right) \rightarrow \ldots
\end{gathered}
$$

and the fact that the extension splits implies that this long exact sequence breaks into short exact sequences

$$
0 \rightarrow H^{i}\left(\Gamma_{\kappa_{\nu}}, \mathbb{Z} / p\right) \rightarrow H^{i}\left(\Gamma_{K_{\nu}}, \mathbb{Z} / p\right) \stackrel{r}{\rightarrow} H^{i-1}\left(\Gamma_{\kappa_{\nu}}, \mathbb{Z} / p\right)
$$

where $r$ can also be explicitly described in terms of cocycles, see GMS], p.16.

In $[\mathrm{B}-\mathrm{P}]$ the following theorem was proven (loc. cit, Theorem 5.1):

Theorem 1.1. There is a natural isomorphism $H_{\mathrm{s}}^{*}\left(\mathfrak{A}_{2 n+1}, \mathbb{Z} / 2 \mathbb{Z}\right) \simeq$ $H_{\mathrm{s}}^{*}\left(\mathfrak{A}_{2 n}, \mathbb{Z} / 2 \mathbb{Z}\right)$, and as a $\mathbb{Z} / 2 \mathbb{Z}$-vector space

$$
H_{\mathrm{s}}^{*}\left(\mathfrak{A}_{2 n}, \mathbb{Z} / 2 \mathbb{Z}\right)=\bigoplus_{0 \leq i \leq n} \mathbb{Z} / 2 \mathbb{Z} \cdot w_{2 i} \oplus \underset{0<i \leq n}{\bigoplus} \mathbb{Z} / 2 \mathbb{Z} \cdot u_{1} \wedge w_{2 i}
$$

where $w_{j}$ are the (images in stable cohomology of) the Stiefel-Whitney classes in $H^{j}\left(\mathfrak{A}_{2 n}, \mathbb{Z} / 2 \mathbb{Z}\right)$ obtained from the cohomology ring of the real orthogonal group $\mathrm{O}(2 n)$ via the inclusions $\mathfrak{A}_{2 n} \subset \mathfrak{S}_{2 n} \subset \mathrm{O}(2 n)$. The class $u_{1}$ is a one-dimensional cohomology class which can be described as follows:

Putting $N=2 n$, the group $\mathfrak{S}_{N}$ acts generically freely on the complement $\mathbb{C}^{N-1}-H$ of the braid hyperplane arrangement $H$ in the standard permutation representation $\mathbb{C}^{N-1}$, and $\left(\mathbb{C}^{N-1}-H\right) / \mathfrak{S}_{N} \simeq \mathbb{C}^{N-1}-\Delta$, the complement of the discriminant. Taking a nonramified double cov-

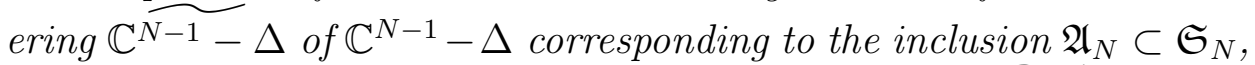
one gets a description of $u_{1}$ as the generator of $H^{1}\left(\widetilde{\mathbb{C}^{N-1}-\Delta}\right)$ given by the root of the discriminant. 
In our Theorem 3.6 we determine $H_{\mathrm{s}}^{*}\left(\mathfrak{A}_{N}, \mathbb{Z} / p \mathbb{Z}\right)$ completely for odd primes $p$.

We base our approach to the computation of the stable cohomology of alternating groups on the following lemmas.

Lemma 1.2. Suppose a group is a product $G \times A$ of finite groups $G$ and $A$ with $A$ abelian. Then there is the Künneth decomposition

$$
H_{\mathrm{s}}^{n}(G \times A, \mathbb{Z} / p) \simeq \bigoplus_{i+j=n} H_{\mathrm{s}}^{i}(G, \mathbb{Z} / p) \otimes H_{\mathrm{s}}^{j}(A, \mathbb{Z} / p)
$$

Proof. It is known ( Bogo93, Lemma 7.1) that if we choose a free presentation $\pi: \mathbb{Z}^{n} \rightarrow A$ of $A$, then the kernel of the stabilization map coincides with the kernel of $\pi^{*}$. In other words, if one realizes $\mathbb{Z}^{n}$ as the fundamental group of some algebraic torus $T$, with cover $T^{\prime} \rightarrow T \simeq T^{\prime} / A$ corresponding to $A$, and realizes $T^{\prime}$ as a maximal torus in some $\mathrm{GL}(W)$, then stabilization is achieved by considering the image of the cohomology of $A$ in the cohomology of $T \simeq T^{\prime} / A \subset$ $W^{L} / A$. This is so because one can find a product of circles $\left(S^{1}\right)^{m}$ in the complement of any divisor $D$ in $T \simeq\left(\mathbb{C}^{*}\right)^{m}$, the inclusion being a homotopy equivalence, so the cohomology of $T$ is already stable. The product of circles can be found by induction on the dimension $m$ of $T$; if $m=1$, one chooses a circle in the complex plane $\mathbb{C}$ not passing through the finite number of points which $D$ consists of. If $m>1$, one views $T$ as a subset of $W$, which is stratified into torus orbits of lower dimension. Each of these is isomorphic to an algebraic tori. Choose a codimension 1 torus orbit $T_{1}$ adjacent to $T$. The closure $\bar{D}$ inside $W$ of a divisor $D \subset T$ meets $T_{1}$ in a proper algebraic subset, and by the induction hypothesis there is a real submanifold $M \simeq\left(S^{1}\right)^{m-1}$ in the complement of $\bar{D} \cap T_{1}$. If $x_{1}, \ldots, x_{m}$ are coordinates in $W$ such that $T=\left\{x_{i} \neq 0 \forall i\right\}, T_{1}=\left\{x_{1}=0 \wedge x_{j} \neq 0 \forall j \neq 1\right\}$, then $W=W^{\prime} \oplus \mathbb{C}$ where $W^{\prime}=\left\{x_{1}=0\right\}$. If we choose a small circle $S_{\epsilon} \subset \mathbb{C}$, then $M \times S_{\epsilon} \subset W$ will be in a small neighbourhood of $M$ hence will not intersect $D$.

This argument can be made relative: note first that there is always a natural surjection $H_{\mathrm{s}}^{*}(G, \mathbb{Z} / p) \otimes H_{\mathrm{s}}^{*}(A, \mathbb{Z} / p) \rightarrow H_{\mathrm{s}}^{*}(G \times A, \mathbb{Z} / p)$ as the Zariski topology on a product is finer than the product topology, and to show it is an isomorphism, it suffices to note the following: suppose $T \simeq\left(\mathbb{C}^{*}\right)^{m} \simeq T^{\prime} / A$ is as before, and $V$ is a generically free $G$-representation, $V^{L}$ the open part where the action is free. Then if $D \subset\left(V^{L} / G\right) \times T$ is any divisor, there is always a divisor $D^{\prime} \subset V^{L} / G$ 
and a relatively compact subset $U^{L} / G \subset V^{L} / G-D^{\prime}$ with a (trivial) iterated circle fibration $U^{L} / G \times\left(S^{1}\right)^{m} \subset\left(\left(V^{L} / G\right) \times T\right)-D$ such that $U^{L} / G \times\left(S^{1}\right)^{m}$ and $\left(\left(V^{L} / G\right) \times T\right)-D$ are homotopy equivalent.

Indeed, viewing $V^{L} / G \times T \subset V^{L} / G \times W$, the latter being a vector bundle, we have a zero section $V^{L} / G \subset V^{L} / G \times W$. Moreover, $\bar{D} \cap$ $V^{L} / G$, where $\bar{D}$ is the closure of $D$ in $V^{L} / G \times W$, will be contained in some divisor $D^{\prime}$. Shrinking $V^{L} / G-D^{\prime}$ slightly, we can find a relatively compact open subset $U^{L} / G \subset V^{L} / G-D^{\prime}$ homotopy equivalent to $V^{L} / G-D^{\prime}$ and with the claimed circle fibration.

We say that the stable cohomology $H_{\mathrm{s}}^{*}(G, \mathbb{Z} / p)$ is detected by abelian subgroups if the map induced by the restriction to abelian subgroups

$$
H_{\mathrm{s}}^{*}(G, \mathbb{Z} / p \mathbb{Z}) \longrightarrow \prod_{A} H_{\mathrm{s}}^{*}(A, \mathbb{Z} / p \mathbb{Z})
$$

is injective (where $A$ ranges over all abelian subgroups of $G$ ). We will then also use the following principle which follows from Lemma 1.2 .

Lemma 1.3. Suppose $G_{1}$ and $G_{2}$ are finite groups such that at least one of $H_{\mathrm{s}}^{*}\left(G_{1}, \mathbb{Z} / p\right)$ or $H_{\mathrm{s}}^{*}\left(G_{2}, \mathbb{Z} / p\right)$ is detected by abelian subgroups. Then one has a Künneth formula in stable cohomology

$$
H_{\mathrm{s}}^{*}\left(G_{1} \times G_{2}, \mathbb{Z} / p\right) \simeq H_{\mathrm{s}}^{*}\left(G_{1}, \mathbb{Z} / p\right) \otimes H_{\mathrm{s}}^{*}\left(G_{2}, \mathbb{Z} / p\right) .
$$

Proof. There is always the natural surjection

$$
H_{\mathrm{s}}^{*}\left(G_{1}, \mathbb{Z} / p\right) \otimes H_{\mathrm{s}}^{*}\left(G_{2}, \mathbb{Z} / p\right) \stackrel{p}{\rightarrow} H_{\mathrm{s}}^{*}\left(G_{1} \times G_{2}, \mathbb{Z} / p\right) .
$$

Without loss of generality, we can assume that abelian subgroups $A_{i}$ are a detecting family for the stable cohomology of $G_{1}$ :

$$
H_{\mathrm{s}}^{*}\left(G_{1}, \mathbb{Z} / p\right) \hookrightarrow \prod_{i \in I} H_{\mathrm{s}}^{*}\left(A_{i}, \mathbb{Z} / p\right) .
$$

Now by Lemma 1.2, there is an injection

$$
H_{\mathrm{s}}^{*}\left(G_{1}, \mathbb{Z} / p\right) \otimes H_{\mathrm{s}}^{*}\left(G_{2}, \mathbb{Z} / p\right) \stackrel{i}{\hookrightarrow} \prod_{i} H_{\mathrm{s}}^{*}\left(A_{i} \times G_{2}, \mathbb{Z} / p\right)
$$

But $i=\left(\prod \operatorname{res}_{A_{i} \times G_{2}}\right) \circ p$ where

$$
\prod \operatorname{res}_{A_{i} \times G_{2}}: H_{\mathrm{s}}^{*}\left(G_{1} \times G_{2}, \mathbb{Z} / p\right) \rightarrow \prod_{i} H_{\mathrm{s}}^{*}\left(A_{i} \times G_{2}, \mathbb{Z} / p\right)
$$

is the product of restriction maps. Hence $p$ is also injective.

Lemma 1.4. Let $G$ be a finite group such that $H_{\mathrm{nr}}^{i}(G, \mathbb{Z} / p \mathbb{Z})=0$ for all $i>0$. Then every stable class $a \in H_{\mathrm{s}}^{*}(G, \mathbb{Z} / p \mathbb{Z})$ is nontrivial on the centralizer $C(g)$ of some element $g \in G$, i.e. the restriction res : $H_{\mathrm{s}}^{*}(G, \mathbb{Z} / p \mathbb{Z}) \rightarrow H_{\mathrm{s}}^{*}(C(g), \mathbb{Z} / p \mathbb{Z})$ is nonzero. 
Proof. With the notation established above we have the maps of groups

$$
I \subset \operatorname{Gal}\left(K_{\nu}\right) \subset \operatorname{Gal}(K) \rightarrow G,
$$

and the image of the inertia subgroup $I$ in $G$ is cyclic, generated by $g$ say, and the image of the decomposition group $\operatorname{Gal}\left(K_{\nu}\right)$ in $G$ belongs to the centralizer $C(g)$. As the residue map $\partial_{\nu}$ factors over $\operatorname{Gal}\left(K_{\nu}\right)$, we obtain the assertion.

Recall the exact sequence

$$
1 \rightarrow I \rightarrow \Gamma_{K_{\nu}} \rightarrow \Gamma_{\kappa_{\nu}} \rightarrow 1
$$

where $I$ is the inertia subgroup of the decomposition group $\Gamma_{K_{\nu}}$ associated to the valuation $\nu$ of $K=\mathbb{C}(V)^{G}$. The following Lemma allows one to increase the usefulness of Lemma 1.4 in inductive arguments further.

Lemma 1.5. Let $G$ be a finite group and let $a \in H_{\mathrm{s}}^{*}(G, \mathbb{Z} / p)$ be a stable class. For $\nu$ a divisorial valuation of $K$, the image of the topologically cyclic inertia subgroup $I$ in $G$ is cyclic, generated by $h$ say. There is a natural class $d_{\nu}(a) \in H_{\mathrm{s}}^{n-1}\left(Z_{G}(h), \mathbb{Z} / p\right)$ such that the residue $\partial_{\nu}(a) \in H^{n-1}\left(\Gamma_{\kappa_{\nu}}, \mathbb{Z} / p\right)$ is the pull-back of $d_{\nu}(a)$ to $\Gamma_{\kappa_{\nu}}$ via the maps $\Gamma_{\kappa_{\nu}} \subset \Gamma_{K_{\nu}} \simeq I \oplus \Gamma_{\kappa_{\nu}} \rightarrow Z(h)$.

Proof. Consider the commutative diagram

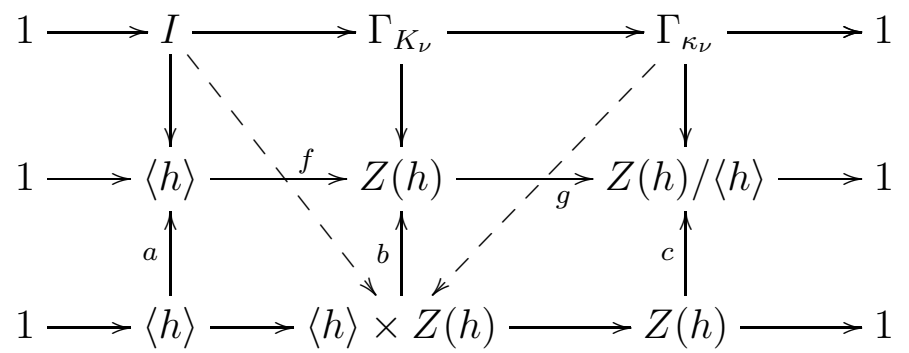

where the arrow $a$ is the identity, $b$ is the couple $\left(i_{\langle h\rangle}, \operatorname{id}_{Z(h)}\right)$ where $i_{\langle h\rangle}:\langle h\rangle \rightarrow Z(h)$ is the inclusion, and $c$ is the projection. Here $f$ and $g$ are defined as follows: the extension defining $\Gamma_{K_{\nu}}$ splits, $\Gamma_{K_{\nu}} \simeq \hat{\mathbb{Z}} \oplus \Gamma_{\kappa_{\nu}}$, so the map $\Gamma_{\kappa_{\nu}} \rightarrow Z(h) /\langle h\rangle$ lifts to a map $g_{1}$ : $\Gamma_{\kappa_{\nu}} \rightarrow Z(h)$. The map $g$ is simply $\left(1, g_{1}\right)$. The map $f$ is $\left(f_{1}, 1\right)$ where $f_{1}: I \rightarrow\langle h\rangle$ is the natural map. As $\hat{\mathbb{Z}}$ has cohomological dimension one, $H^{i}\left(\Gamma_{K_{\nu}}, \mathbb{Z} / p\right) \simeq H^{i-1}\left(\Gamma_{\kappa_{\nu}}, \mathbb{Z} / p\right) \oplus H^{i}\left(\Gamma_{\kappa_{\nu}}, \mathbb{Z} / p\right)$ and the residue map $\partial_{\nu}$ was defined by the restriction of $a$ to $\Gamma_{K_{\nu}}$ and projecting to $H^{i-1}\left(\Gamma_{\kappa_{\nu}}, \mathbb{Z} / p\right)$. By the commutativity of the diagram, we may thus define a class $d_{\nu}(a)$ with the requested properties as follows: we restrict $a \in H_{\mathrm{s}}^{n}(G, \mathbb{Z} / p)$ to $Z(h)$, and then take the pull-back 
$b^{*}\left(\operatorname{res}_{Z(h)}^{G}(a)\right) \in H_{\mathrm{s}}^{n}(\langle h\rangle \times Z(h), \mathbb{Z} / p)$ and project this unto the component $H_{\mathrm{s}}^{n-1}(Z(h), \mathbb{Z} / p)$ in the Künneth decomposition, using Lemma 1.2. This defines $d_{\nu}(a) \in H_{\mathrm{s}}^{n-1}(Z(h), \mathbb{Z} / p)$.

Define a subgroup $H$ of $G$ recursively to be an iterated centralizer if it is the centralizer of an element in $G$ or a centralizer of an element inside another iterated centralizer.

Corollary 1.6. Assume that $G$ is such that each iterated centralizer has trivial unramified cohomology. Then any element $a \in H_{\mathrm{s}}^{n}(G, \mathbb{Z} / p)$ is nontrivial on some abelian p-subgroup.

Proof. We use induction on the cohomological degree, hence assume that every element in $H_{\mathrm{s}}^{i}(H, \mathbb{Z} / p)$, for all $i<n$, and for all iterated centralizers $H$ in $G$, is nontrivial on some abelian subgroup. By assumption, we get from Lemma 1.5 that $d_{\nu}(a) \in H_{\mathrm{s}}^{n-1}(Z(h), \mathbb{Z} / p)$ is nontrivial for some $h$. Hence $d_{\nu}(a)$ is nontrivial on some abelian $p$ subgroup $A$ of $Z(h)$. By the construction of $d_{\nu}(a)$ in Lemma 1.5 we have that $a$ will then be nontrivial when restricted to $H_{\mathrm{s}}^{n}(\langle h, A\rangle, \mathbb{Z} / p)$ where $\langle h, A\rangle$ is the abelian subgroup of $G$ generated by $h$ and $A$.

The Steenrod power operations $S q^{i}, P^{j}$ (see [Steen, A-M II.2) are natural transformations

$$
\begin{gathered}
S q^{i}: H^{j}(X, \mathbb{Z} / 2) \rightarrow H^{j+i}(X, \mathbb{Z} / 2), \\
P^{i}: H^{j}(X, \mathbb{Z} / p) \rightarrow H^{j+2 i(p-1)}(X, \mathbb{Z} / p), p \text { an odd prime },
\end{gathered}
$$

on the category of CW-complexes with continuous maps $f: X \rightarrow Y$. By functoriality, applied to the map BG $\rightarrow\left(V^{L} / G\right)-D$, where $D$ is some divisor, $S q^{i}, P^{j}$ induce operations on $H_{\mathrm{s}}^{*}(G, \mathbb{Z} / p)$.

For later use, we recall here the structure theorem for the cohomology of wreath products due to Steenrod [Steen], Section VII, see also Mann78, Theorem 3.1 and [A-M], IV. 4, Theorem 4.1. We suppress the $\mathbb{Z} / p \mathbb{Z}$-coefficients in cohomology groups now, i.e. write $H^{*}(X)$ for $H^{*}(X, \mathbb{Z} / p \mathbb{Z})$.

Theorem 1.7. Let $H$ be a group, and let $H \backslash \mathbb{Z} / p=(H)^{p} \rtimes \mathbb{Z} / p$ be the wreath product where $\mathbb{Z} / p \mathbb{Z}$ acts by cyclically permuting the copies of $H$. Let $\mathrm{id} \times \Delta^{p}: \mathbb{Z} / p \mathbb{Z} \times H \rightarrow \mathbb{Z} / p \ltimes(H)^{p}$ be the inclusion $\left(\mathrm{id} \times \Delta^{p}\right)(z, a)=$ $(z ;(a, \ldots, a))$ (p-times $a)$ and denote by $t: H^{*}\left(H^{p}\right) \rightarrow H^{*}(H \prec \mathbb{Z} / p)$ the transfer. Then the sequence

$$
\left.H^{*}\left(H^{p}\right) \stackrel{t}{\rightarrow} H^{*}(H<\mathbb{Z} / p) \stackrel{\left(\mathrm{id} \times \Delta^{p}\right.}{\longrightarrow}\right)^{*} H^{*}(\mathbb{Z} / p \mathbb{Z} \times H)
$$


is exact.

Moreover, for any $u \in H^{j}(H)$ there is a class $P(u) \in H^{j p}(H \prec \mathbb{Z} / p)$ (constructed by Steenrod) such that

(i) If $j: H^{p} \rightarrow \mathbb{Z} / p \mathbb{Z} \ltimes(H)^{p}$ is the natural inclusion, then $j^{*}(P(u))=$ $u^{\otimes p}$.

(ii) In the Künneth decomposition of $\left(\mathrm{id} \times \Delta^{p}\right)^{*}(P(u))$ in $H^{*}(\mathbb{Z} / p \mathbb{Z} \times$ $H)$ we have

$$
\left(\mathrm{id} \times \Delta^{p}\right)^{*}(P(u))=\Sigma w_{k} \otimes D_{k}(u)
$$

where $w_{k}$ is a generator of $H^{k}(\mathbb{Z} / p \mathbb{Z})$ and $D_{k}: H^{q}(H) \rightarrow$ $H^{p q-k}(H)$ are homomorphisms which satisfy

(iii)

$$
\beta D_{2 k}(u)=D_{2 k-1}(u), \beta D_{2 k-1}(u)=0, \beta D_{0}(u)=0
$$

where $\beta$ is the Bockstein homomorphism, i.e. connecting homomorphism in the long exact sequence coming from the short exact sequence

$$
0 \rightarrow \mathbb{Z} / p \mathbb{Z} \rightarrow \mathbb{Z} / p^{2} \mathbb{Z} \rightarrow \mathbb{Z} / p \mathbb{Z} \rightarrow 0
$$

The maps $D_{k}$ are used originally by Steenrod to define the Steenrod powers $P^{i}$, hence the Hopf algebras (Steenrod algebras) $\mathcal{A}(p)$. More precisely,

$$
P^{i}(u)=(-1)^{i+m j(j+1) / 2}(m !)^{j} D_{(p-1)(j-2 i)}(u) \in H^{j+2(p-1) i}(H, \mathbb{Z} / p \mathbb{Z}) .
$$

In this setting, any $c \in H^{*}(H<\mathbb{Z} / p)$ can be written as

$$
c=t\left(c_{1}\right)+c_{2} \cdot P\left(c_{3}\right)
$$

with $c_{1} \in H^{*}\left(H^{p}\right), c_{2} \in H^{*}(\mathbb{Z} / p \mathbb{Z})$ and $c_{3} \in H^{*}(H)$.

Here we view $H^{*}(H<\mathbb{Z} / p)$ as a module over $H^{*}(\mathbb{Z} / p \mathbb{Z})$ via the cohomology pull-back induced by the surjection $H<\mathbb{Z} / p \rightarrow \mathbb{Z} / p \mathbb{Z}$. The Steenrod operations $P^{i}$ have the following formal properties:

(1) $P^{0}=\mathrm{id}$.

(2) If $\operatorname{dim}(x)=2 n$, then $P^{n}(x)=x^{p}$.

(3) If $2 i>\operatorname{dim}(x)$, then $P^{i}(x)=0$.

(4) (Cartan formula) $P^{i}(x \cup y)=\sum_{j=0}^{i} P^{j}(x) \cup P^{i-j}(y)$.

A consequence of the Bloch-Kato conjecture (now a theorem by the work of Voevodsky, Rost and many others) is

Lemma 1.8. The Steenrod cohomology operations $S q^{i}, P^{j}$ are zero in stable cohomology $H_{\mathrm{s}}^{*}(G, \mathbb{Z} / p \mathbb{Z})$. 
Proof. Any stable class $a \in H_{\mathrm{s}}^{*}(G, \mathbb{Z} / p \mathbb{Z}) \subset H^{*}(\operatorname{Gal}(K), \mathbb{Z} / p)$ arises as the pull-back from some torus $T \simeq\left(\mathbb{C}^{*}\right)^{m}$, more precisely, a has a representative $a^{\prime}$ in the cohomology of $H^{*}\left(\left(V^{L} / G\right)-D, \mathbb{Z} / p\right)$ and there is a regular map $f:\left(V^{L} / G\right)-D \rightarrow T$ together with a class $\hat{a} \in H^{*}(T, \mathbb{Z} / p)$ with $f^{*}(\hat{a})=a^{\prime}$. This follows from the fact, which is a consequence of the Bloch-Kato conjecture, that $H^{*}\left(\mathrm{Gal}^{\mathrm{ab}}(K), \mathbb{Z} / p\right) \rightarrow$ $H^{*}(\mathrm{Gal}(K), \mathbb{Z} / p)$ is surjective where $\mathrm{Gal}^{\mathrm{ab}}(K)$ is the abelianized Galois group $\operatorname{Gal}(K) /[\operatorname{Gal}(K), \operatorname{Gal}(K)]$.

Since the Steenrod power operations are trivial in the cohomology algebra of the torus $T$ (which is an exterior algebra on one-dimensional generators), the assertion of the Lemma will then follow from the functoriality of the cohomology operations.

It remains to explain in some more detail how from the surjection $H^{*}\left(\mathrm{Gal}^{\mathrm{ab}}(K), \mathbb{Z} / p\right) \rightarrow H^{*}(\operatorname{Gal}(K), \mathbb{Z} / p)$ we get the map $f:\left(V^{L} / G\right)-$ $D \rightarrow T$. There is a finite abelian quotient $\operatorname{Gal}(K) \rightarrow A$ such that $a$ is induced from a class $a^{\prime \prime}$ in the cohomology of $A$. The group $A$ corresponds to an unramified abelian covering $\tilde{X}$ of some nonempty open affine subvariety $X \subset V^{L} / G$. The coordinate ring $\mathbb{C}[\tilde{X}]$ contains an arbitrary finite dimensional representation of $A$ as the regular functions on an $A$-orbit in $\tilde{X}$ are precisely the regular representation $\mathbb{C}[A]$ and this is also a subrepresentation (not only a quotient) because $A$ is reductive. In particular, embedding $A$ in a torus of diagonal matrices in some $\mathrm{GL}_{m}(\mathbb{C})$, one obtains a dominant regular map from an open subset $X^{\prime}$ of $X=\tilde{X} / A$, hence some $\left(V^{L} / G\right)-D$ to a torus $T=\left(\mathbb{C}^{m}\right)^{L} / A \simeq\left(\mathbb{C}^{*}\right)^{m}$ for which it holds by construction that the image of the class $a^{\prime \prime}$ in the cohomology of $T$ induces a representative $a^{\prime} \in H^{*}\left(\left(V^{L} / G\right)-D, \mathbb{Z} / p\right)$ of $a$.

Thus the techniques used in the present article are mainly topological in flavour; for the connection to motivic cohomology and further developments the reader may consult [Kahn-Su00, [Kahn11, Ngu1, Ngu2, [TY11].

\section{Detection By elementary abelian $p$-Subgroups}

In this section we want to prove the following Theorem.

Theorem 2.1. Let $p$ as always be an odd prime and $\mathfrak{A}_{N}$ the alternating group on $N$ letters. Then $H_{\mathrm{s}}^{*}\left(\mathfrak{A}_{N}, \mathbb{Z} / p\right)$ is detected by elementary abelian p-subgroups.

We denote by $G_{n}=\mathbb{Z} / p \mathbb{Z} \imath \cdots \imath \mathbb{Z} / p \mathbb{Z}$ ( $n$ factors) the iterated wreath product of $n$ cyclic $p$-groups. This is the $p$-Sylow of $\mathfrak{A}_{p^{n}}$. If $N$ is 
arbitrary (not necessarily a power of $p$ ) expand it in base $p$ :

$$
N=a_{0}+a_{1} p+\cdots+a_{m} p^{m}
$$

with $0 \leq a_{j}<p, a_{m} \neq 0$, and note that this gives rise to a natural inclusion

$$
i_{a_{1}, \ldots, a_{m}}: \mathfrak{A}_{a_{1}, \ldots, a_{m}}:=\prod_{1}^{a_{1}} \mathfrak{A}_{p} \times \prod_{1}^{a_{2}} \mathfrak{A}_{p^{2}} \times \cdots \times \prod_{1}^{a_{m}} \mathfrak{A}_{p^{m}} \hookrightarrow \mathfrak{A}_{N}
$$

and that a $p$-Sylow subgroup in $\mathfrak{A}_{N}$ is given by the product of $p$-Sylow subgroups in the factors in $\mathfrak{A}_{a_{1}, \ldots, a_{m}}$. Hence it follows from Lemma 1.3 that it is enough to prove Theorem 2.1 for $N=p^{n}$.

First we will prove the weaker

Theorem 2.2. The stable cohomology $H_{\mathrm{s}}^{*}\left(\mathfrak{A}_{N}, \mathbb{Z} / p\right)$ is detected by abelian p-subgroups.

The proof of Theorem 2.2 will follow from the structures of centralizers in complete monomial groups.

Definition 2.3. Let $H$ be a group. The complete monomial group of degree $m$ on $H$ is the group $\Sigma_{m}(H):=H$ ᄀ $\mathfrak{S}_{m}=(H)^{m} \rtimes \mathfrak{S}_{m}$, where $\mathfrak{S}_{m}$ is the symmetric group on $m$ letters.

A monomial cycle in $\Sigma_{m}(H)$ is an element of the form $\left(\left(h_{1}, \ldots, h_{m}\right) ; \sigma\right)$ where $\sigma \in \mathfrak{S}_{m}$ is a cycle. The determinant class of a monomial cycle is the conjugacy class in $H$ of the product $h_{1} \cdot \ldots \cdot h_{m}$. The length of a monomial cycle is the length of the underlying cycle $\sigma$.

We have to recall some results from Ore on the structure of conjugacy classes and centralizers in groups $\Sigma_{m}(H)$ :

(1) Two monomial cycles in $\Sigma_{m}(H)$ are conjugate if and only if they have the same length and determinant class ([Ore], Theorem 6).

(2) Any element $x \in \Sigma_{m}(H)$ can be written uniquely as a product of commuting monomial cycles (the underlying cyles in $\mathfrak{S}_{m}$ have no common variables) ([Ore, Theorem 3).

(3) From (1) and (2) follows the description of conjugacy classes in $\Sigma_{m}(H)$ : two elements $x^{\prime}, x$ in $\Sigma_{m}(H)$ are conjugate if the monomial cycles in their decompositions in (2) can be matched in such a way that corresponding cycles have the same length and determinant class.

(4) Let $c=((h, \ldots, 1,1) ; \sigma)$ be a monomial cycle of length $m$ in $\Sigma_{m}(H)$. Then its centralizer in $\Sigma_{m}(H)$ is an extension

$$
1 \rightarrow Z_{H}(h) \rightarrow Z_{\Sigma_{m}(H)}(c) \rightarrow \mathbb{Z} / m \rightarrow 0
$$


(Ore, p.20, 21). In other words, the centralizer $Z_{\Sigma_{m}(H)}(c)$ is generated by $c$ and the group $Z_{H}(h)$ embedded diagonally into $H^{m} \subset \Sigma_{m}(H)$.

(5) ([Ore, Theorem 8): Let

$$
x=x_{1} \cdot \ldots \cdot x_{l}, x_{i}=y_{1}^{(i)} \cdot \ldots \cdot y_{k_{i}}^{(i)}
$$

be the decomposition of an element $x$ in $\Sigma_{m}(H)$ into disjoint monomial cycles $y_{j}^{(i)}$ as in (2), where we group the cycles of equal length and determinant class together: for fixed $i$, all $y_{j}^{(i)}$ have determinant class $h_{i}$ and length $n_{i}$. Then the centralizer of $x$ in $\Sigma_{m}(H)$ has a description as

$$
Z_{\Sigma_{m}(H)}(x)=\prod_{i=1}^{l} \Sigma_{k_{i}}\left(Z_{\Sigma_{n_{i}}(H)}\left(y_{1}^{(i)}\right)\right)
$$

where as in (4) the centralizer of $y_{1}^{(i)}$ (or any $y_{j}^{(i)}$ ) in the group $\Sigma_{n_{i}}(H)$ is an extension

$$
1 \rightarrow Z_{H}\left(h_{i}\right) \rightarrow Z_{\Sigma_{n_{i}}(H)}\left(y_{1}^{(i)}\right) \rightarrow \mathbb{Z} / n_{i} \mathbb{Z} \rightarrow 0
$$

From the last fact (5) we get immediately

Lemma 2.4. Let $\Sigma_{m}(A)$ be a complete monomial group with $A$ abelian. Then the centralizer of any element $x$ in $\Sigma_{m}(A)$ is a product of groups of the same type

$$
Z_{\Sigma_{m}(A)}(x)=\prod_{h=1}^{M} \Sigma_{k_{h}}\left(A_{h}\right)
$$

where the $A_{h}$ are abelian.

Proof. It suffices to remark that any central extension of a cyclic group by an abelian group is again abelian.

To prove Theorem 2.2 it suffices, by the technique of Lemma 1.5 exposed above, to show the following.

Lemma 2.5. Let $p$ be an odd prime and let $x$ be an element of order a power of $p$ in a group $A \mathfrak{A}_{m}=A^{m} \rtimes \mathfrak{A}_{m}$ where $A$ is an abelian p-group. Then there is a group

$$
Z^{\prime}=\prod_{h=1}^{M} A_{h} \prec \mathfrak{A}_{k_{h}}
$$

where all the $A_{h}$ are abelian p-groups, and with the property that $Z^{\prime}$ is contained in the centralizer $Z_{A \mathfrak{A}_{m}}(x)$ and contains a $p$-Sylow of $Z_{A \nmid \mathfrak{A}_{m}}(x)$. 
Proof. We consider the group $A<\mathfrak{A}_{m}$ as a subgroup of the complete monomial group $\Sigma_{m}(A)$. By Lemma 2.4 itsuffices to determine the intersection of $Z_{\Sigma_{m}(A)}(x)=\prod_{h=1}^{M} \Sigma_{k_{h}}\left(A_{h}\right)$ and $A<\mathfrak{A}_{m}$. As $p$ is odd, it is clear that the intersection contains

$$
Z^{\prime}=\prod_{h=1}^{M} A_{h} \prec \mathfrak{A}_{k_{h}}
$$

and that the complete centralizer $Z_{A \backslash \mathfrak{A}_{m}}(x)$ is an extension

$$
1 \rightarrow Z^{\prime} \rightarrow Z_{A i \mathfrak{A}_{m}}(x) \rightarrow(\mathbb{Z} / 2)^{r} \rightarrow 0
$$

where $(\mathbb{Z} / 2)^{r}$ is an elementary abelian 2-group which can be identified with the kernel of the sign

$$
\prod_{h=1}^{M} \mathfrak{S}_{k_{h}} \subset \mathfrak{S}_{\sum k_{h}} \rightarrow\{ \pm 1\}
$$

modulo the subgroup $\prod_{h=1}^{M} \mathfrak{A}_{k_{h}}$. The statement follows as $p$ is odd.

Thus we obtain

Proof. (of Theorem 2.2) We will prove more generally that $H_{\mathrm{s}}^{*}(G, \mathbb{Z} / p$ ) is detected by abelian $p$-subgroups where $G$ is any group which is a product of groups $A<\mathfrak{A}_{m}$ with $A$ an abelian $p$-group. We have that $H_{\mathrm{s}}^{*}(G, \mathbb{Z} / p)$ is detected by $H_{\mathrm{s}}^{*}\left(\operatorname{Syl}_{p}(G), \mathbb{Z} / p\right)$, and the higher unramified cohomology of $\operatorname{Syl}_{p}(G)$ is trivial. This follows immediately from B-P], Lemma 2.4, namely, if one forms a wreath product of groups, each of which has stably rational generically free linear quotients, then the wreath product inherits this property.

Hence every element $a$ in $H_{\mathrm{s}}^{n}(G, \mathbb{Z} / p)$ will, in the notation of Lemma 1.5, give a nontrivial $d_{\nu}(a) \in H_{\mathrm{s}}^{n-1}\left(Z_{G}(h), \mathbb{Z} / p\right)$ for some element $h \in G$ of $p$-power order. Thus it will be enough to show that $H_{\mathrm{s}}^{n-1}\left(Z_{G}(h), \mathbb{Z} / p\right)$ is detected by abelian $p$-subgroups. But $H_{\mathrm{s}}^{n-1}\left(Z_{G}(h), \mathbb{Z} / p\right)$ is detected by $H_{\mathrm{s}}^{n-1}\left(\operatorname{Syl}_{p}\left(Z_{G}(h)\right), \mathbb{Z} / p\right)$ and, by Lemma 2.5 , $\operatorname{Syl}_{p}\left(Z_{G}(h)\right)$ is contained in a group which in turn is contained in $Z_{G}(h)$ and is again a product of groups of type $A<\mathfrak{A}_{m}$. Hence we can conclude by induction on the cohomological degree $n$.

Now we prove Theorem 2.1, It will follow immediately from

Proposition 2.6. Let $N=p^{n}$, and suppose that $A$ is an abelian $p$ subgroup of $\mathfrak{A}_{N}$. Thus one can write

$$
A=\prod_{i=1}^{k}\left(\mathbb{Z} /\left(p^{l_{i}}\right)\right)^{r_{i}}, l_{i}, r_{i} \in \mathbb{N} .
$$


If $A$ is not reduced to a single cyclic group $\mathbb{Z} /\left(p^{l}\right)$, then $A$ is contained in a product of alternating groups $\prod_{j=1}^{h} \mathfrak{A}_{t_{h}} \subset \mathfrak{A}_{N}$ with $t_{h}<N$ for all $h$.

Once we have this Proposition, the proof of Theorem 2.1 is an induction: it suffices to prove it for $N=p^{n}$, and we may assume that detection by elementary abelian subgroups holds for the stable cohomology of all $\mathfrak{A}_{j}$ with $j<N$. Now clearly, $H_{\mathrm{s}}^{1}\left(\mathfrak{A}_{N}, \mathbb{Z} / p\right)$ is detected by elementary abelian $p$-subgroups, for $H^{1}\left(\mathfrak{A}_{N}, \mathbb{Z} / p\right)$ can be identified with characters $\chi: \mathfrak{A}_{N} \rightarrow \mathbb{Z} / p$ whence $H_{\mathrm{s}}^{1}\left(\mathfrak{A}_{N}, \mathbb{Z} / p\right)=0$ unless $p=3$ and $N=3$ so that $\mathfrak{A}_{N}=\mathbb{Z} / 3$. So we have to show that any stable class $a \in H_{\mathrm{s}}^{i}\left(\mathfrak{A}_{N}, \mathbb{Z} / p\right)$ for $i \geq 2$ is nontrivial on an elementary abelian $p$-subgroup. By Theorem $2.2 a$ is nontrivial on an abelian $p$-subgroup $A$ with $\operatorname{rk}(A / p A) \geq 2$ (as the stable cohomology of $A$ is an exterior algebra on $\operatorname{rk}(A / p A)$ generators). Such an $A$ is contained in a product of smaller alternating groups by Proposition 2.6. Thus the proof is complete by induction.

Proof. (of Proposition 2.6)

Denote by $X_{N}=\{1, \ldots, N\}$ the set of letters on which the ambient $\mathfrak{S}_{N} \supset A$ acts. Let

$$
X_{N}=\coprod_{\alpha} X_{N, \alpha}
$$

be the decomposition of $X_{N}$ into $A$-orbits. Let $X_{N, \alpha_{0}}=: X$ be a fixed orbit. This orbit is isomorphic to a quotient $\bar{A}$ of $A$, hence a group of the same form

$$
\bar{A}=\prod_{i=1}^{\bar{k}}\left(\mathbb{Z} /\left(p^{\bar{l}_{i}}\right)\right)^{\bar{r}_{i}}
$$

and the action of $A$ on this orbit is via the regular representation of $\bar{A}$ on itself. In other words, $A$ embeds into a subgroup $\prod_{\alpha} \bar{A}_{\alpha}$ of $\mathfrak{A}_{N}$ where each $\bar{A}_{\alpha}$ is embedded into a subgroup $\mathfrak{A}_{\text {ord }\left(\bar{A}_{\alpha}\right)}$ via the regular representation.

In summary, it suffices to prove the statement of Proposition 2.6 for the case that the group $A$ in its statement is embedded into the ambient $\mathfrak{A}_{N}$ via the regular representation. We can write $A=A^{\prime} \times \mathbb{Z} /\left(p^{k}\right)$ with $\operatorname{rk}\left(A^{\prime} / p A^{\prime}\right)<\operatorname{rk}(A / p A)$. Moreover, by definition of the regular representation, the composition of arrows

$$
A=A^{\prime} \times \mathbb{Z} /\left(p^{k}\right) \hookrightarrow \mathfrak{A}_{\left|A^{\prime}\right|} \times \mathbb{Z} /\left(p^{k}\right) \hookrightarrow \mathfrak{A}_{\left|A^{\prime}\right|}\left\langle\mathbb{Z} /\left(p^{k}\right) \hookrightarrow \mathfrak{A}_{\left|A^{\prime}\right| \cdot p^{k}} \simeq \mathfrak{A}_{|A|}\right.
$$

gives the regular representation of $A$ where the first arrow $\hookrightarrow$ from the left is induced by the regular representation of $A^{\prime}$, the second such arrow embeds $\mathfrak{A}_{\left|A^{\prime}\right|} \times \mathbb{Z} /\left(p^{k}\right)$ into the wreath product $\mathfrak{A}_{\left|A^{\prime}\right|}\left\langle\mathbb{Z} /\left(p^{k}\right)\right.$ by 
sending $(a ; \sigma)$ to $(a, a, \ldots, a ; \sigma)$ as usual, and the last arrow embeds the wreath product $\mathfrak{A}_{\left|A^{\prime}\right|}\left\langle\mathbb{Z} /\left(p^{k}\right)\right.$ into $\mathfrak{A}_{\left|A^{\prime}\right| \cdot p^{k}}$ by partitioning the set of $\left|A^{\prime}\right| \cdot p^{k}$ objects which $\mathfrak{A}_{\left|A^{\prime}\right| \cdot p^{k}}$ permutes into $p^{k}$ disjoint groups of $\left|A^{\prime}\right|$ objects, and letting $\mathbb{Z} /\left(p^{k}\right)$ act by cyclically rotating these groups, and letting $\left(\mathfrak{A}_{\left|A^{\prime}\right|}\right)^{p^{k}}$ act via permutations within these groups. It follows that

$$
A \subset \mathfrak{A}_{\left|A^{\prime}\right|} \times \mathfrak{A}_{p^{k}}
$$

where now $\mathfrak{A}_{p^{k}}$ is embedded into $\mathfrak{A}_{|A|}$ as arbitrary alternating (not only cyclic) permutations of the $p^{k}$ groups of items. Note that elements of the two subgroups $\mathfrak{A}_{p^{k}}$ and $\mathfrak{A}_{\left|A^{\prime}\right|}$ of the group $\mathfrak{A}_{|A|}$ commute, and the two subgroups intersect trivially, so that we do have a direct product. Moreover, if $A$ is not reduced to a single cyclic group, we have that $A^{\prime}$ is not the trivial group, and $p^{k}<|A|$.

\section{Stable Cohomology of alternating Groups}

Let $\mathfrak{A}_{n}$ be, as in the previous section, the alternating group on $n$ letters, and let $p$ be an odd prime (the case $p=2$ has been treated in $[\mathrm{B}-\mathrm{P}]$ ). We assume first $n=p^{m}$ for simplicity.

We have to know the way elementary abelian $p$-subgroups sit inside $\mathfrak{A}_{n}$ for the following. We summarize everything in the following Lemma which is proven by arguments analogous to those already used in the proof of Proposition 2.6.

Lemma 3.1. Suppose $n=p^{m}$ and denote by $I_{m}:=\left\{\underline{i}=\left(i_{1}, \ldots, i_{m}\right) \in\right.$ $\left.\mathbb{N}^{m}\right\}$ the set of all nonnegative integer sequences $\underline{i}$ with

$$
p^{m}=i_{1} p+i_{2} p^{2}+\cdots+i_{m} p^{m}=\sum_{j=1}^{m} i_{j} p^{j} .
$$

Then there is a natural bijection between $I_{m}$ and the set of conjugacy classes of maximal elementary abelian p-subgroups in $\mathfrak{S}_{p^{m}}$. The subgroup $T\left(i_{1}, \ldots, i_{m}\right)$ corresponding to $\underline{i}$ can be described as follows: partition the set of integers $X=\{1, \ldots, n\}$ into segments of $p$ power lengths according to $\underline{i}$ :

$$
X=\bigcup_{j=1}^{m} \bigcup_{s=1}^{i_{j}} X_{s}^{j}
$$

where $X_{s}^{j}$ is a set with $p^{j}$ elements, $X_{s}^{j}=\left\{i_{1} p+\cdots+i_{j-1} p^{j-1}+(s-1) p^{j}, \ldots, i_{1} p+\cdots+i_{j-1} p^{j-1}+s p^{j}\right\}$ 
for definiteness. The subset $X_{s}^{j}$ corresponds to a subgroup $\mathfrak{S}_{p^{j}}=$ $\left(\mathfrak{S}_{p^{j}}\right)^{X_{s}^{j}} \subset \mathfrak{S}_{p^{m}}$ fixing all elements in $X$ outside $X_{s}^{j}$. Inside $\left(\mathfrak{S}_{p^{j}}\right)^{X_{s}^{j}}$ there is a copy of $(\mathbb{Z} / p \mathbb{Z})^{j}$, which we denote by $\left((\mathbb{Z} / p \mathbb{Z})^{j}\right)^{X_{s}^{j}}$, embedded via the regular representation, i.e. we identify the elements in $X_{s}^{j}$ with the elements of $\left((\mathbb{Z} / p \mathbb{Z})^{j}\right)^{X_{s}^{j}}$ and the permutation action is then given by left multiplication.

We denote $T\left(0, \ldots, p^{m-k}, \ldots, 0\right)$ (a single nonzero entry $p^{m-k}$ in the $k$-th place) by $T_{k, m}$.

Hence every maximal elementary abelian p-subgroup in $\mathfrak{A}_{n}$ is conjugate -in $\mathfrak{S}_{n}$ or $\mathfrak{A}_{n}$, it is the same thing- to one contained in $\mathfrak{A}_{p^{n-1}} \times \cdots \times \mathfrak{A}_{p^{n-1}}$ ( $p$ factors) or conjugate to $T_{m, m}$.

The proof is immediate if one notices that under the action of some elementary abelian $p$-subgroup $A$ the set $X$ breaks up into $A$ orbits of cardinality a $p$ power, and the action of $A$ restricted to an orbit embeds $A$ into the permutation group of the elements of the orbit in such a way that the image is conjugate to the image of the regular representation. The result is in A-M VI. 1, Thm. 1.3, but also [Mui], Chapter II, §2, where it is ascribed to Dixon. For the statement that the conjugacy classes of maximal elementary abelian $p$-subgroups in $\mathfrak{A}_{n}$ are the same as in $\mathfrak{S}_{n}$ one can appeal to the following Lemma which we will also use in other instances below (it is e.g. in Mann85, p. 269).

Lemma 3.2. For $n=p^{m}$ the Weyl groups $W_{\mathfrak{S}_{n}}\left(T_{m, m}\right)=N_{\mathfrak{S}_{n}}\left(T_{m, m}\right) / T_{m, m}$ resp. $W_{\mathfrak{A}_{n}}\left(T_{m, m}\right)$ of $T_{m, m} \simeq(\mathbb{Z} / p \mathbb{Z})^{m}$ inside $\mathfrak{S}_{n}$ resp. $\mathfrak{A}_{n}$ are

$$
W_{\mathfrak{S}_{n}}\left(T_{m, m}\right)=\mathrm{GL}_{m}\left(\mathbb{F}_{p}\right), W_{\mathfrak{A}_{n}}\left(T_{m, m}\right)=\mathrm{GL}_{m}^{+}\left(\mathbb{F}_{p}\right)
$$

where $\mathrm{GL}_{m}^{+}\left(\mathbb{F}_{p}\right)$ is the kernel of the map $\mathrm{GL}_{m}\left(\mathbb{F}_{p}\right) \rightarrow \mathbb{Z} / 2 \mathbb{Z}$ given by the determinant raised to the power $(p-1) / 2$.

In fact it is true that the Weyl group of any group $H$ in the embedding $H \hookrightarrow \mathfrak{S}_{|H|}$ given by the regular representation is the group of outer automorphisms of $H$, which become all inner in $\mathfrak{S}_{|H|}$. Both statements of the Lemma follow from this remark as $\operatorname{Aut}\left((\mathbb{Z} / p \mathbb{Z})^{m}\right)=\operatorname{GL}\left(m, \mathbb{F}_{p}\right)$. Likewise, Lemma 3.2 implies that in the normalizer of any maximal elementary abelian $p$-subgroup in $\mathfrak{S}_{n}$ there are elements which do not lie in $\mathfrak{A}_{n}$. Hence conjugacy classes of these in the two groups coincide.

We will also use in an essential way the Cárdenas-Kuhn Theorem to calculate the stable cohomology of $\mathfrak{A}_{n}$, so we recall the precise statement (see $[\mathrm{A}-\mathrm{M}]$ III.5 for the proof). 
Theorem 3.3. Let $E \varsubsetneqq S \varsubsetneqq G$ be a closed system of finite groups, where the closedness means that every subgroup of $S$ which is conjugate to $E$ in $G$ is already conjugate to $E$ in $S$. Let $W_{G}(E)=N_{G}(E) / E$ resp. $W_{S}(E)=N_{S}(E) / E$ be the Weyl groups of $E$ in $G$ resp. $S$, and suppose that $E$ is p-elementary and that $W_{S}(E)$ contains a p-Sylow of $W_{G}(E)$. Then the image of the restriction map

$$
\operatorname{res}_{E}^{G}: H^{*}(G, \mathbb{Z} / p \mathbb{Z}) \rightarrow H^{*}(E, \mathbb{Z} / p \mathbb{Z})
$$

is equal to

$$
\operatorname{im}\left(\operatorname{res}_{E}^{S}: H^{*}(S, \mathbb{Z} / p \mathbb{Z}) \rightarrow H^{*}(E, \mathbb{Z} / p \mathbb{Z})\right) \cap H^{*}(E, \mathbb{Z} / p \mathbb{Z})^{W_{G}(E)} .
$$

We will mostly use this in the form of the following

Corollary 3.4. Let $S$ be a p-Sylow of a finite group $G$, and let $E$ be an elementary abelian p-subgroup of $S$. Suppose that any subgroup of $S$ conjugate to $E$ in $G$ is conjugate to $E$ in $S$. Then we have

$$
\begin{gathered}
\operatorname{im}\left(\operatorname{res}_{E}^{G}: H^{*}(G, \mathbb{Z} / p \mathbb{Z}) \rightarrow H^{*}(E, \mathbb{Z} / p \mathbb{Z})\right) \\
=\operatorname{im}\left(\operatorname{res}_{E}^{S}: H^{*}(S, \mathbb{Z} / p \mathbb{Z}) \rightarrow H^{*}(E, \mathbb{Z} / p \mathbb{Z})\right) \cap H^{*}(E, \mathbb{Z} / p \mathbb{Z})^{W_{G}(E)} .
\end{gathered}
$$

Proof. It suffices to remark that $[G: S] \equiv\left[N_{G}(E): N_{S}(E)\right] \not \equiv 0(\bmod$ $p)$.

Now if $n$ is arbitrary (not necessarily a power of $p$ ), to understand $H_{\mathrm{s}}^{*}\left(\mathfrak{A}_{n}, \mathbb{Z} / p \mathbb{Z}\right)$, expand $n$ in base $p$ :

$$
n=a_{0}+a_{1} p+\cdots+a_{m} p^{m}
$$

with $0 \leq a_{j}<p, a_{m} \neq 0$, and note that this gives rise to a natural inclusion

$$
i_{a_{1}, \ldots, a_{m}}: \mathfrak{A}_{a_{1}, \ldots, a_{m}}:=\prod_{1}^{a_{1}} \mathfrak{A}_{p} \times \prod_{1}^{a_{2}} \mathfrak{A}_{p^{2}} \times \cdots \times \prod_{1}^{a_{m}} \mathfrak{A}_{p^{m}} \hookrightarrow \mathfrak{A}_{n}
$$

and that a $p$-Sylow subgroup in $\mathfrak{A}_{n}$ is given by the product of $p$-Sylow subgroups in the factors in $\mathfrak{A}_{a_{1}, \ldots, a_{m}}$. In the notation of Lemma 3.1, the group $\mathfrak{A}_{a_{1}, \ldots, a_{m}}$ contains an elementary abelian $p$-subgroup

$$
E:=\prod_{1}^{a_{1}} T_{1,1} \times \prod_{1}^{a_{2}} T_{1,2} \times \cdots \times \prod_{1}^{a_{m}} T_{1, m} \simeq(\mathbb{Z} / p \mathbb{Z})^{\frac{n-a_{0}}{p}} .
$$

Proposition 3.5. The group $E$ detects the stable cohomology of $\mathfrak{A}_{n}$, i.e.

$$
H_{\mathrm{s}}^{*}\left(\mathfrak{A}_{n}, \mathbb{Z} / p \mathbb{Z}\right) \rightarrow H_{\mathrm{s}}^{*}(E, \mathbb{Z} / p \mathbb{Z})
$$

is injective. 
Proof. It will be sufficient to prove this for $n=p^{m}$ as a Künneth theorem holds in stable cohomology for groups whose stable cohomology is detected by abelian subgroups, cf. Lemma 1.3. Now $\mathfrak{A}_{p^{m}}$ contains the wreath product

$$
\mathfrak{A}_{p^{m-1}}<\mathbb{Z} / p \mathbb{Z}
$$

which detects the stable cohomology of $\mathfrak{A}_{p^{m}}$ as it contains a $p$-Sylow. Using induction, it will be sufficient to prove that

$$
H_{\mathrm{s}}^{*}\left(\mathfrak{A}_{p^{m}}, \mathbb{Z} / p \mathbb{Z}\right) \rightarrow H_{\mathrm{s}}^{*}\left(\mathfrak{A}_{p^{m-1}} \times \cdots \times \mathfrak{A}_{p^{m-1}}\right)
$$

is injective for $m>1$. By Lemma 3.1 and because $H_{s}^{*}\left(\mathfrak{A}_{n}, \mathbb{Z} / p \mathbb{Z}\right)$ is detected by elementary abelian $p$-subgroups, it will be sufficient to show that all positive-dimensional classes in $H^{*}\left(T_{m, m}, \mathbb{Z} / p \mathbb{Z}\right)$ coming as restrictions from $H^{*}\left(\mathfrak{A}_{p^{m}}, \mathbb{Z} / p \mathbb{Z}\right)$ are unstable. This follows from the calculation in [Mann85], Theorem 1.9, and the fact that the Bocksteins are zero in stable cohomology.

Theorem 3.6. Let $p$ be an odd prime as before. Then $H_{\mathrm{s}}^{*}\left(\mathfrak{A}_{n}, \mathbb{Z} / p \mathbb{Z}\right)=$ 0 in positive degrees unless $p=3$. For $p=3$ one has for $k \in \mathbb{N}$

$$
H_{\mathrm{s}}^{*}\left(\mathfrak{A}_{3 k}, \mathbb{Z} / 3 \mathbb{Z}\right) \simeq H_{\mathrm{s}}^{*}\left(\mathfrak{A}_{3 k+1}, \mathbb{Z} / 3 \mathbb{Z}\right), \quad H_{\mathrm{s}}^{d}\left(\mathfrak{A}_{3 k+2}, \mathbb{Z} / 3 \mathbb{Z}\right)=0, d>0,
$$

and

$$
\begin{gathered}
H_{\mathrm{s}}^{d}\left(\mathfrak{A}_{3 k}, \mathbb{Z} / 3 \mathbb{Z}\right) \neq 0 \text { for } d>0 \Longleftrightarrow d=k \text {, and } \\
H_{\mathrm{s}}^{k}\left(\mathfrak{A}_{3 k}, \mathbb{Z} / 3 \mathbb{Z}\right) \simeq\left\langle\operatorname{det}_{k}\right\rangle \text { where } \operatorname{res}_{E}^{\mathfrak{A}_{3 k}}\left(\operatorname{det}_{k}\right)=e_{1} \wedge \cdots \wedge e_{k}
\end{gathered}
$$

where $H_{\mathrm{s}}^{*}(E, \mathbb{Z} / 3 \mathbb{Z})=H_{\mathrm{s}}^{*}\left((\mathbb{Z} / 3 \mathbb{Z})^{k}, \mathbb{Z} / 3 \mathbb{Z}\right)$ is an exterior algebra on one-dimensional generators $e_{1}, \ldots, e_{k}$.

Basically, we would like to use the Cardénas-Kuhn Theorem with the elementary abelian subgroup $E$, and $S=\operatorname{Syl}_{p}\left(\mathfrak{A}_{n}\right), G=\mathfrak{A}_{n}$, but it will be more transparent to break it up into several steps.

Lemma 3.7. For $p \neq 3$ an odd prime we have in positive degrees $H_{\mathrm{s}}^{*}\left(\mathfrak{A}_{n}, \mathbb{Z} / p \mathbb{Z}\right)=0$.

Proof. The Weyl group $W_{\mathfrak{A}_{n}}(E)$ contains two obvious subgroups: (1) the group $\mathfrak{A}_{N}$ permuting the $N:=\left(n-a_{0}\right) / p$ copies of $\mathbb{Z} / p \mathbb{Z}$ in $E$, (2) a product $\prod_{1}^{N}(\mathbb{Z} / p \mathbb{Z})^{*,+}$ where $(\mathbb{Z} / p \mathbb{Z})^{*,+}$ is the subgroup of the group of units in $\mathbb{Z} / p \mathbb{Z}$ given as the kernel of $a \mapsto a^{(p-1) / 2}$. The stable cohomology of $E$ is an exterior algebra over $\mathbb{Z} / p \mathbb{Z}$ on $N$ generators $e_{1}, \ldots, e_{N}$. The $\mathfrak{A}_{N}$-invariants are concentrated in degrees $0,1,(N-$ 1), $N$, one-dimensional in each case and generated by

$$
1, \quad e_{1}+\cdots+e_{N}, \quad f_{1} \wedge \cdots \wedge f_{N-1}, \quad e_{1} \wedge \cdots \wedge e_{N}
$$


where $f_{1}, \ldots, f_{N-1}$ is a basis of the $\mathfrak{A}_{N}$-invariant complement to $e_{1}+$ $\cdots+e_{N}$ in $H^{1}(E, \mathbb{Z} / p \mathbb{Z})$. All of these are not invariant under the scalings in $\prod_{1}^{N}(\mathbb{Z} / p \mathbb{Z})^{*,+}$ unless $p=3$ when $(\mathbb{Z} / p \mathbb{Z})^{*,+}$ is reduced to $\{1\}$.

Lemma 3.8. One has

(1) $H_{\mathrm{s}}^{d}\left(\mathfrak{A}_{3 k+2}, \mathbb{Z} / 3 \mathbb{Z}\right)=0, d>0$.

(2) There is a natural embedding

$$
H_{\mathrm{S}}^{*}\left(\mathfrak{A}_{3 k+1}, \mathbb{Z} / 3 \mathbb{Z}\right) \hookrightarrow H_{\mathrm{s}}^{*}\left(\mathfrak{A}_{3 k}, \mathbb{Z} / 3 \mathbb{Z}\right) .
$$

Proof. This is already contained in $\mathrm{B}-\mathrm{P}$, Lemmas 4.1 and 4.2. For completeness, let us repeat the argument: the restriction $\operatorname{res}_{\operatorname{Syl}_{3}\left(\mathfrak{A}_{3 k+2}\right)}^{\mathfrak{A}_{3 k+2}}$ factors through the restriction map induced from the embedding $\mathfrak{S}_{3 k} \hookrightarrow$ $\mathfrak{A}_{3 k+2}$; but $H_{\mathrm{s}}^{*}\left(\mathfrak{S}_{3 k}, \mathbb{Z} / 3 \mathbb{Z}\right)=0$ in positive degrees as the stable cohomology of $\mathfrak{S}_{3 k}$ is detected by its elementary abelian 2-subgroup generated by a maximal set of commuting transpositions. This proves (1), and (2) follows from the fact that the 3-Sylows in $\mathfrak{A}_{3 k}$ and $\mathfrak{A}_{3 k+1}$ are the same.

Lemma 3.9. Let $n=3 k$ or $n=3 k+1$. Then the Weyl group $N_{\mathfrak{A}_{n}}(E)$ of $E \simeq(\mathbb{Z} / 3 \mathbb{Z})^{k}$ in $\mathfrak{A}_{n}$ sits in an extension

$$
1 \rightarrow(\mathbb{Z} / 2 \mathbb{Z})^{k-1} \rightarrow W_{\mathfrak{A}_{n}}(E) \rightarrow \mathfrak{S}_{k} \rightarrow 1
$$

where $\mathfrak{S}_{k}$ acts by permuting the copies of $\mathbb{Z} / 3 \mathbb{Z}$ in $E \simeq(\mathbb{Z} / 3 \mathbb{Z})^{k}$, and the group $(\mathbb{Z} / 2 \mathbb{Z})^{k-1}$ acts by sending an even number of the generators $g_{i}$ in the ith copy of $\mathbb{Z} / 3 \mathbb{Z}$ to their inverses $g_{i}^{-1}$. In stable cohomology $H_{\mathrm{s}}^{*}(E, \mathbb{Z} / 3 \mathbb{Z})=E\left(e_{1}, \ldots, e_{k}\right)$ (exterior algebra), the action of the group $W_{\mathfrak{A}_{n}}(E)$ is generated by the (signed) transpositions sending $e_{i}, e_{j}$ to $e_{j},-e_{i}$, and transformations corresponding to elements in $(\mathbb{Z} / 2 \mathbb{Z})^{k-1}$ acting via sign changes $e_{i} \mapsto-e_{i}$ on an even number of the $e_{i}$.

Proof. Any element of the normalizer $N_{\mathfrak{A}_{n}}(E)$ induces a well-defined permutation of the copies of $\mathbb{Z} / 3 \mathbb{Z}$ in $E$. This gives a map to $\mathfrak{S}_{k}$ which is onto: note that conjugating the 3-cycle (123) by $\tau=(23)$ exchanges the two nontrivial elements $g, g^{-1}$ in $\mathbb{Z} / 3 \mathbb{Z}=\langle(123)\rangle$, so we can also transpose two copies of $\mathbb{Z} / 3 \mathbb{Z}$ in $E$ by conjugating by an element in $\mathfrak{A}_{n}$. Now suppose $n \in N_{\mathfrak{A}_{n}}(E)$ induces the trivial element in $\mathfrak{S}_{k}$, so fixes all the copies of $\mathbb{Z} / 3 \mathbb{Z}$ in $E$ (though not necessarily elementwise). Then the only possible nontrivial automorphism of each copy of $\mathbb{Z} / 3 \mathbb{Z}$ is exchanging $g$ and $g^{-1}$ as before. To conclude the proof, it suffices to note that if $n$ induces the identity in $\operatorname{Aut}\left((\mathbb{Z} / 3 \mathbb{Z})^{k}\right)$, then $n \in(\mathbb{Z} / 3 \mathbb{Z})^{k}$.

We can now turn to the 
Proof. (of Theorem 3.6) The remaining assertion not covered by Lemma 3.7 and Lemma 3.8 are that

$$
\begin{gathered}
H_{\mathrm{s}}^{d}\left(\mathfrak{A}_{3 k}, \mathbb{Z} / 3 \mathbb{Z}\right) \neq 0 \text { for } d>0 \Longleftrightarrow d=k, \text { and } \\
H_{\mathrm{s}}^{k}\left(\mathfrak{A}_{3 k}, \mathbb{Z} / 3 \mathbb{Z}\right) \simeq\left\langle\operatorname{det}_{k}\right\rangle \text { where } \operatorname{res}_{E}^{\mathfrak{A}_{3 k}}\left(\operatorname{det}_{k}\right)=e_{1} \wedge \cdots \wedge e_{k} .
\end{gathered}
$$

and that

$$
H_{\mathrm{s}}^{*}\left(\mathfrak{A}_{3 n+1}, \mathbb{Z} / 3 \mathbb{Z}\right) \rightarrow H_{\mathrm{s}}^{*}\left(\mathfrak{A}_{3 n}, \mathbb{Z} / 3 \mathbb{Z}\right)
$$

is surjective (it is injective by (2) of Lemma 3.8).

We prove first the assertions in the displayed formula 1 above, and 2 will follow easily (we just have to check that the determinant class comes from $\left.H_{\mathrm{s}}^{*}\left(\mathfrak{A}_{3 n+1}, \mathbb{Z} / 3 \mathbb{Z}\right)\right)$. We apply the Cardénas-Kuhn Theorem 3.3 with $S=\operatorname{Syl}_{3}\left(\mathfrak{A}_{3 k}\right)$ containing $E$ and $G=\mathfrak{A}_{3 k}$. Then

- The fact that $E \simeq(\mathbb{Z} / 3 \mathbb{Z})^{k} \subset \operatorname{Syl}_{3}\left(\mathfrak{A}_{3 k}\right) \subset \mathfrak{A}_{3 k}$ is a closed system has been checked in [Mui], Prop. 2.2: in fact, he checks that if $A$ is any maximal elementary abelian $p$-subgroup of a symmetric group $\mathfrak{S}_{n}$, then any subgroup of a $p$-Sylow $\operatorname{Syl}_{p}\left(\mathfrak{S}_{n}\right)$ containing $A$ which is conjugate to $A$ in $\mathfrak{S}_{n}$ is conjugate to $A$ in $\operatorname{Syl}_{p}\left(\mathfrak{S}_{n}\right)$. This implies clearly the statement for the alternating groups we need.

- By the Cardénas-Kuhn Theorem or rather its Corollary 3.4, we get that the image of the cohomology of $\mathfrak{A}_{3 k}$ in the cohomology of $E$ is

$\operatorname{im}\left(\operatorname{res}_{E}^{\operatorname{Syl}_{3}\left(\mathfrak{A}_{3 k}\right)}: H^{*}\left(\operatorname{Syl}_{3}\left(\mathfrak{A}_{3 k}, \mathbb{Z} / 3 \mathbb{Z}\right) \rightarrow H^{*}(E, \mathbb{Z} / p \mathbb{Z})\right) \cap H^{*}(E, \mathbb{Z} / 3 \mathbb{Z})^{W_{\mathfrak{A}_{3 k}}(E)}\right.$.

- By Theorem 1.7 and induction

$$
\operatorname{res}_{E}^{\mathfrak{A}_{3 k}}: H^{*}\left(\mathrm{~A}_{3 k}, \mathbb{Z} / 3 \mathbb{Z}\right) \rightarrow H^{*}(E, \mathbb{Z} / 3 \mathbb{Z})^{W_{\mathfrak{A}_{3 k}}(E)}
$$

is surjective (compare also the argument in [Mui], Prop. 3.9 and Lemma 3.11).

Thus

$$
\operatorname{res}_{E}^{\mathfrak{A}_{3 k}}: H_{\mathrm{s}}^{*}\left(\mathrm{~A}_{3 k}, \mathbb{Z} / 3 \mathbb{Z}\right) \simeq H_{\mathrm{s}}^{*}(E, \mathbb{Z} / 3 \mathbb{Z})^{W_{\mathfrak{A}_{3 k}}(E)}
$$

and by the description of the action of $W_{\mathfrak{A}_{3 k}}$ on the stable cohomology of $E$, we find that only $e_{1} \wedge \cdots \wedge e_{k}$ remains spanning the positive dimensional invariants.

Finally, to prove the surjectivity of the arrow in the displayed formula 2 above, consider the inclusions $E \subset \mathfrak{A}_{3 n} \subset \mathfrak{A}_{3 n+1}$. Then $\operatorname{Syl}_{3}\left(\mathfrak{A}_{3 k}\right)=$ $\mathrm{Syl}_{3}\left(\mathfrak{A}_{3 k+1}\right)$ and, in exact analogy to the argument above, by [Mui], 
Prop. 2.2, $E \subset \operatorname{Syl}_{3}\left(\mathfrak{A}_{3 k+1}\right) \subset \mathfrak{A}_{3 n+1}$ is a closed system, so that by Cardénas-Kuhn the image of the cohomology of $\mathfrak{A}_{3 n+1}$ in the cohomology of $E$ coincides with the image of the cohomology of $\mathfrak{A}_{3 n}$ in $E$ (because also $W_{\mathfrak{A}_{3 n}}(E) \simeq W_{\mathfrak{A}_{3 n+1}}(E)$ ).

\section{REFERENCES}

[A-M] Adem, A., Milgram, R. J., Cohomology of finite groups, 2nd ed., Grundlehren der Mathematischen Wissenschaften 309, SpringerVerlag, Berlin, 2004.

[Ben] Benson, D.J., Representations and Cohomology II. Cohomology of groups and modules, Cambridge studies in advanced mathematics 31, Cambridge University Press (1991)

[Bogo93] Bogomolov, F., Stable Cohomology of Groups and Algebraic Varieties, Russian Acad. Sci. Sb. Math. Vol. 76 (1993), No. 1, 1-21

[Bogo95] Bogomolov, F., On the structure of the Galois groups of the fields of rational functions, Proceedings of Symposia in Pure Mathematics, Volume 58.2 (1995) (S. Barbara conference 1992 "K-theory and quadratic forms") p. 83-88.

[Bogo05] Bogomolov, F., Stable cohomology of finite and profinite groups, in: Algebraic Groups (Yuri Tschinkel (Ed.)), Mathematisches Institut Georg-August-Universität Göttingen Summer School 27.06.13.07.2005, Universitätsverlag Göttingen 2007

[BMP] Bogomolov, F., Maciel, J., Petrov, T., Unramified Brauer groups of finite simple groups of Lie type $A_{l}$, Amer. J. Math. 126 (2004), no. 4 , p. $935-949$

[BPT] Bogomolov, F., Petrov, T., Tschinkel, Yu., Unramified cohomology of finite groups of Lie type, in Cohomological and geometric approaches to rationality problems, Progr. Math., 282, Birkhäuser Boston, Inc., Boston, MA, 2010, p. 5573

[B-P] Bogomolov, F., Petrov, T., Unramified cohomology of alternating groups, Cent. Eur. J. Math. 9 (5), (2011), 936-948

[CTO] Colliot-Thélène, Ojanguren, M., Variétés unirationelles non rationelles: au delà de l'exemple d'Artin et Mumford, Invent. Math. 97 (1989), 141-158

[GMS] Garibaldi, S., Merkurjev, A., Serre, J.-P., Cohomological invariants in Galois cohomology, University Lecture Series 28, American Mathematical Society, Providence, RI, 2003.

[Evens] Evens, L., The Cohomology of Groups, Oxford Mathematical Monographs, Oxford University Press (1991)

[Kahn-Su00] Kahn, Bruno, Sujatha, R., Motivic cohomology and unramified cohomology of quadrics J. European Math. Soc. 2 (2000), 145-177.

[Kahn11] Kahn, Bruno, Relatively unramified elements in cycle modules, J. K-Theory 7 (2011), no. 3, 409?427

[Mann78] Mann, B.M., The cohomology of the symmetric groups, Transactions of the American Mathematical Society Vol. 242 (1978), 157184 
[Mann85] Mann, B.M., The cohomology of the alternating groups, Michigan Math. J. 32 (1985), no. 3, 267277.

[Mui] Mùi, Huỳnh, Modular invariant theory and cohomology algebras of symmetric groups, J. Fac. Sci. Univ. Tokyo Sect. IA Math. 22 (1975), no. 3, 319-369

[Ngu1] Nguyen, Thi Kim Ngan, Modules de cycles et classes non ramifiées sur un espace classifiant, Thèse de Doctorat, dirigée par Bruno Kahn, Université Paris VII-Denis Diderot (2010)

[Ngu2] Nguyen, Thi Kim Ngan, Classes non ramifiées sur un espace classifiant. (French) [Unramified classes over a classifying space] C. R. Math. Acad. Sci. Paris 349 (2011), no. 5-6, 233?237

[Ore] Ore, Oystein, Theory of monomial groups, Trans. Amer. Math. Soc. 51, (1942), 15?64

[Pey08] Peyre, E., Unramified cohomology of degree 3 and Nother's problem, Invent. Math. 171 (2008), 191-225

[Piru] Pirutka, Alena, Cohomologie non ramifiée en degré trois d'une variété de Severi-Brauer. (French. English, French summary) [Degree-three nonramified cohomology of Severi-Brauer varieties] C. R. Math. Acad. Sci. Paris 349 (2011), no. 7-8, 369?373

[Quill71a] Quillen, D., The Adams Conjecture, Topology Vol. 10 (1971), 67-80

[Quill71b] Quillen, D., The spectrum of an equivariant cohomology ring I, Annals of Math. 94 (1971), 549-572

[Quill71c] Quillen, D., The spectrum of an equivariant cohomology ring II, Annals of Math. 94 (1971), 573-602

[Quill78] Quillen, D., Homotopy Properties of the Poset of Nontrivial pSubgroups of a Group, Advances in Math. 28 (1978), 101-128

[Serre] Serre, J-P., Galois Cohomology, Springer Monographs in Mathematics, Springer-Verlag (2002)

[Steen] Steenrod, N.E., Cohomology Operations, Lectures by N.E. Steenrod, written and revised by D.B.A. Epstein, Annals of Mathematics Studies 50, Princeton University Press 1962

[TY11] Tezuka, M. \& Yagita, N., The image of the map from group cohomology to Galois cohomology, Trans. Amer. Math. Soc. 363 (2011), 4475-4503.

F. Bogomolov, Courant Institute of Mathematical Sciences, 251 MerCER St., New York, NY 10012, U.S.A., and

Laboratory of Algebraic Geometry, GU-HSE, 7 Vavilova Str., Moscow, Russia, 117312

E-mail address: bogomolo@courant.nyu.edu

Christian Böhning, Fachbereich Mathematik der Universität Hamburg, Bundesstrasse 55, 20146 Hamburg, Germany

E-mail address: christian.boehning@math.uni-hamburg.de 